\title{
Knowledge Point-Based Approach to Interlink Open Education Resources
}

\author{
Xinglong Ma \\ Design, Engineering \& Computing, Bournemouth University, Poole, UK \\ xma@bournemouth.ac.uk
}

\begin{abstract}
With more and more Open Education Resources (OER) courses being recognised and acknowledged by global learners, an emerging issue is that learners' self-efficacy is often affected by the lack of interaction between peers and instructors in their continuous self-learning process. This paper proposes a low-level Knowledge Point-based approach to serve application layers to enhance the interaction during the self-learning. This is achieved through taking advantage of Semantic Web and Linked Data techniques to annotate and interlink OER fragments which can later be reused and interoperated more conveniently.
\end{abstract}

Keywords: Linked Data, Open Education Resources, Annotation, Knowledge Point, Media Fragment, Self-Learning.

\section{Introduction}

An increasing number of universities and organisations are now participating in carrying forward the development of OER since MIT launched the OpenCourseWare $\left(\mathrm{OCW}^{1}\right)$ initiative in 2001. With the multimedia based OER information (video, audio, digitalised textbooks and documents, etc.), global learner can freely access and schedule their self-learning. However, the process becomes more monotonous and unexciting by missing traditional interactive classroom. This often results that most learners are struggling to catch up with the whole curriculum and complete the course. To address this issue, a Knowledge Point-based approach is proposed to semantically annotate and interlink OER fragments rather than a collection of OER material, which provides the support of flexible reuse and interoperation of OER to serve learning applications.

This paper is organised as follows. First, the research problem is described. Second, current Linked Data and annotation technologies for OER are discussed. Third, the proposed Knowledge Point approach is introduced. Last, the schedule and related methodologies are briefed.

1 http://ocw.mit.edu/index.htm

P. Cimiano et al. (Eds.): ESWC 2013, LNCS 7882, pp. 717-721, 2013.

(C) Springer-Verlag Berlin Heidelberg 2013 


\section{Motivation and Research Questions}

Without giving timely feedback and assessment, online learners often feel less motivated compared to learners in the traditional classroom learning [1, 2]. In terms of OER, massive open online courses (MOOCs), such as Khan Academy ${ }^{2}$ and Coursera ${ }^{3}$, are trying to improve this situation by providing more interactive environment with quizzes during or after class. However, these quizzes, which are usually predefined and arbitrary, cannot be easily reused and interoperated with open access. In most situations, once an OER material is published, no on-going supplements are maintained and served although it is essential for self-learning.

To date, most OER data are collected in distributed repositories, such as OCW, OER Commons ${ }^{4}$, Merlot ${ }^{5}$, where data are annotated by different metadata mechanisms (e.g. IEEE $\mathrm{LOM}^{6}, \mathrm{ADL} \mathrm{SCORM}^{7}$ ) and retrieved by individual web APIs/services [3].

This PhD project will research on how to reuse and interoperate isolated OER and in which way these OER can be more openly and flexibly accessed to promote interaction in self-learning.

\section{State of the Art}

Semantic web and Linked Data technologies have recently been exploited and applied into the field of the technology enhanced learning (TEL) to improve the learning performance and enable the reuse and interoperation of OER data. Following the Linked Data principles [4], URIs are used to name the OER data, which can be unambiguously identified. With the aid of URIs, the corresponding OER data and relevant interlinked data can be dereferenced, which are machine-readable and repurposed to serve the dynamic assessment system to enhance interaction with reused and interoperated OER data.

In [5-7], researchers presented Linked Data based approaches to automatically and dynamically generate learning assessments via DBpedia ${ }^{8}$, publishing Wikipedia information on the web. However, these approaches do not reuse existing huge and diverse OER data. Instead, they highly rely on the knowledge from Wikipedia.

On the other hand, OER provided with Linked data facilitates the process of reuse and interoperation, which has been implemented by a few universities, such as Open

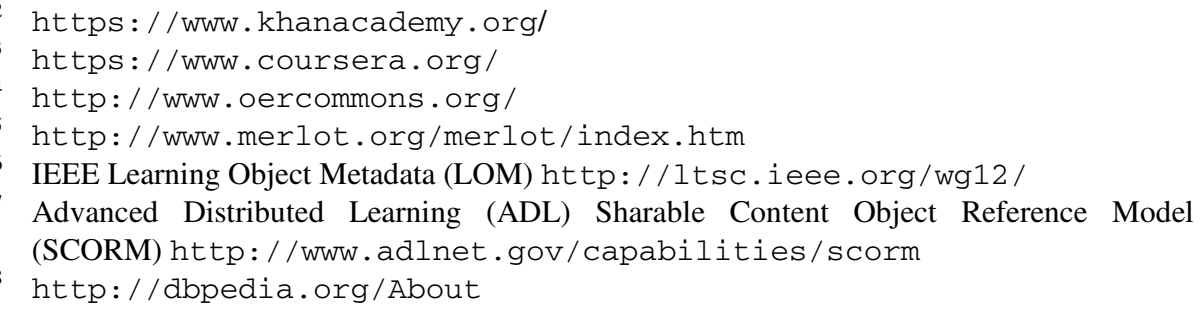


University ${ }^{9}$, University of Southampton ${ }^{10}$ and University of Oxford ${ }^{11}$. However, most OER data are still distributed in different OER repositories. Then a lot of researches have been done to integrate and generalise the metadata, such as OAI-PMH ${ }^{12}$, which are registered by 1888 repositories [8], and ontology-based approaches[9-11]. However, these technologies are limited because the annotated data cannot be dereferenced [12] and these annotations are for a block or collection of OER rather than a single or certain part of OER material. Therefore more advanced technologies are required to enrich the OER data with Linked Data, such as textual analysis, text mining, information extraction and natural languages processing (NLP).

In another way, the annotation can be clinged to the certain section of an individual OER material to avert NLP kind of complex approaches, which can be implemented for videos and audios by using Media Fragments ${ }^{13}$. In [13], it applies the Media Fragments and NERD ${ }^{14}$ to annotate the YouTube video fragments with Linked Data. Yet, for OER, there are more types of open data.

\section{$4 \quad$ Proposed Approach}

In this section, it proposes the concept of Knowledge Point (KP) and an annotation approach that refines annotation granularity and is based on the LOD Cloud to semantically annotate variety of OER materials.

In Fig. 1, the left-side shows the distributed and heterogeneous OER Repositories, which store multifarious of OER data, such as videos, documents, etc. After processing "Knowledge Point Annotation", the single material wrapped in a block or collection can be virtually "cut into multiple fragments". And a single fragment can be annotated by more than one KP which are acquired or extracted from manifold datasets of LOD Cloud ${ }^{15}$. Based on the KP Annotation mechanism, the RDF with Linked Data can be used in conjunction with higher-level educational applications.

Characteristics of KP include:

- Fully Compliant to best practice: As a member of web of data, KP complies with the Linked Data principles.

- Independent and atomy: KP can be operated independently and will not be affected by other KP.

- On-demand Fragmentation: An individual OER material can be fragmentised on the basis of KP and an OER fragment can be attributed by manifold KPs.

- KP and OER fragments intersupplement: KP annotates the OER fragment while the OER fragment explains the KP.

\footnotetext{
9 http: //data.open.ac.uk

10 http://data.soton.ac.uk

11 http: //data.ox.ac.uk

12 Open Archives Initiative - Protocol for Metadata Harvesting http: / / www. openarchives.org/pmh/

13 Media Fragments URI 1.0 http: / / www.w3 .org/TR/media-frags /

14 Named Entity Recognition and Disambiguation http: / / nerd. eurecom. fr/

15 Linked Open Data Cloud http: / / Iinkeddata.org/
} 


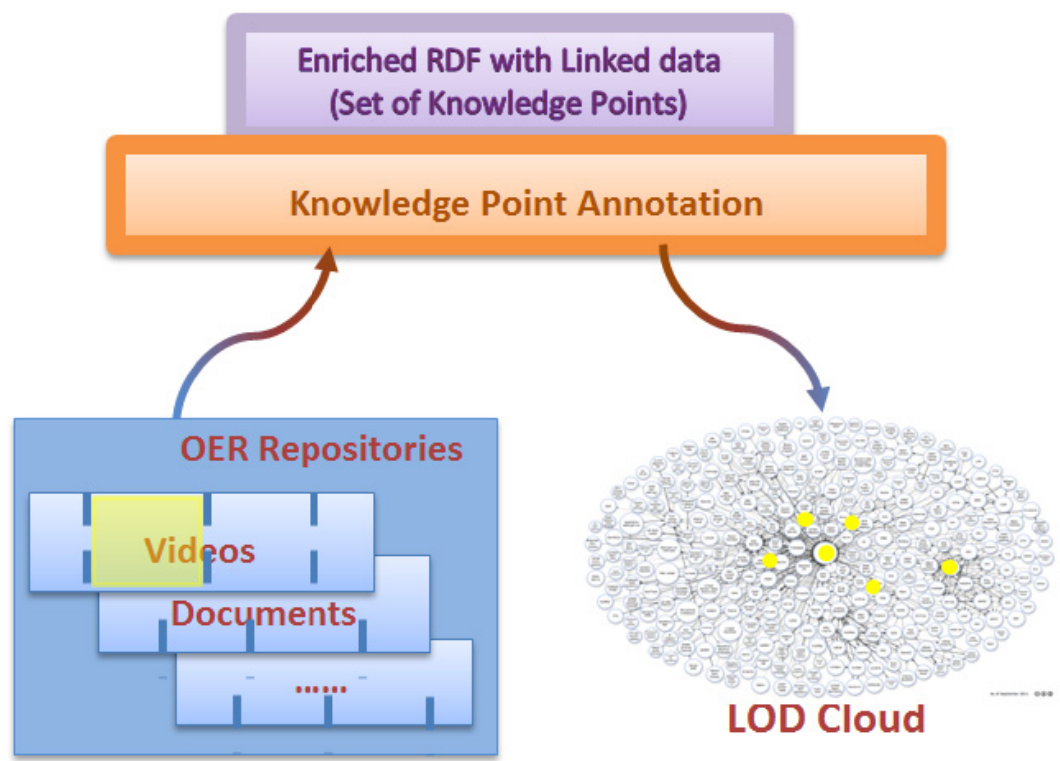

Fig. 1. Knowledge Point-Based Approach to Interlink OER (Left-side OER are virtually cut into multiple fragments. Left certain fragment with yellow can be annotated by the right-side yellow knowledge points distributed over LOD Cloud.)

There are a few challenges that could be seen in this proposed approach which also need to be address in the development. For example, accessing a single file over the distributed OER repositories and blocked file conveniently; compating all OER formats; extracting suitable KPs from LOD Cloud and fragmentising the OER data automatically and precisely.

\section{Scheduled Methodology}

- January 2013 - January 2014:Propose a lightweight mechanism to describe and annotate the heterogeneous OER in fragment level

- January - April: Review the currently lightweight annotation technologies (e.g. Media Fragment URI ${ }^{16}$, Tagging, Folksonomy, etc.) and mainstream OER repositories tools and technologies (e.g. DSpace ${ }^{17}$, Eprints ${ }^{18}$, OAI-PMH, etc.).

- April - June: Find a possible approach on how to annotate the diverse OER materials distributed in different repositories which provide different access methods.

- June - October: Prototype this approach and test its efficiency and performance.

- September - January 2014: Evaluate this approach and improve it.

\footnotetext{
16 http: //www.w3.org/TR/media-frags/

17 http: //www.dspace.org/

18 http://www.eprints.org/
} 
- February 2014 - July 2014: Research on how to extract KPs from general lexical resources (e.g. DBpedia) and domain-specific ones (e.g. GeoNames ${ }^{19}$ ).

- July 2014 - November 2014: Using the proposed KPs extraction mechanism to extend the above prototype.

- October 2014 - May2015: Repeat testing, evaluating and improving to enhance this KP-based approach.

- September 2015: Finish and submit the PhD dissertation.

\section{References}

1. Rovai, A.P., Barnum, K.T.: On-line course effectiveness: An analysis of student interactions and perceptions of learning. The Journal of Distance Education/Revue de l'Éducation à Distance 18, 57-73 (2007)

2. Gikandi, J.W., Morrow, D., Davis, N.E.: Online formative assessment in higher education: A review of the literature. Comput. Educ. 57, 2333-2351 (2011)

3. Dietze, S., Sanchez, S., Ebner, H., Yu, H.Q., Giordano, D., Marenzi, I., Nunes, B.P.: Interlinking educational Resources and the Web of Data - a Survey of Challenges and Approaches. Electronic Library and Information Systems 47 (2013)

4. Berners-Lee: Design Issues, http: / / www.w3 .org/DesignIssues / LinkedData.html

5. Foulonneau, M.: Generating educational assessment items from linked open data: The case of dBpedia. In: García-Castro, R., Fensel, D., Antoniou, G. (eds.) ESWC 2011. LNCS, vol. 7117, pp. 16-27. Springer, Heidelberg (2012)

6. Bratsas, C., Kontokostas, D., Eftychiadou, A., Kontokostas, D., Bamidis, P., Antoniou, I.: Semantic Web Game Based Learning: An I18n approach with Greek DBpedia. In: 2nd International Workshop on Learning and Education with the Web of Data, Lyon, France, April 17 (2012)

7. Waitelonis, J., Ludwig, N., Knuth, M., Sack, H.: WhoKnows? Evaluating linked data heuristics with a quiz that cleans up DBpedia. Interactive Technology and Smart Education 8, 236-248 (2011)

8. Open Archives Initiative:

http: / /www . openarchives.org/Register/BrowseSites

9. Mikroyannidis, A., Lefrere, P., Scott, P.: An Architecture for Layering and Integration of Learning Ontologies, Applied to Personal Learning Environments and Cloud Learning Environments. In: 2010 IEEE 10th International Conference on Advanced Learning Technologies (ICALT), pp. 92-93 (2010)

10. Piedra, N., Chicaiza, J., Lopez, J., Martinez, O., Caro, E.T.: An approach for description of Open Educational Resources based on semantic technologies. In: 2010 IEEE Education Engineering (EDUCON), pp. 1111-1119 (2010)

11. Rocha Amorim, R., Rabelo, T., Amorim, D.: Open Educational Resources Ontology. In: II International Symposium on OER: Issues for Globalization and Localization (2012)

12. Haslhofer, B., Schandl, B.: The OAI2LOD Server: Exposing OAI-PMH metadata as linked data. In: Proceedings of WWW 2008 Workshop Linked Data on the Web (2008)

13. Li, Y., Rizzo, G., Troncy, R., Wald, M., Wills, G.: Creating enriched YouTube media fragments With NERD using timed-text. In: 11th International Semantic Web Conference, November 11-15 (2012)

19 http: //www.geonames.org/ 